\title{
Turbulence and Magnetic Fields in the Large Scale Structure of the Universe
}

\author{
Dongsu Ryu, ${ }^{1 *}$ Hyesung Kang, ${ }^{2}$ Jungyeon Cho, ${ }^{1}$ and Santabrata Das ${ }^{3}$ \\ ${ }^{1}$ Department of Astronomy and Space Science, Chungnam National University, \\ Daejeon 305-764, Korea \\ ${ }^{2}$ Department of Earth Sciences, Pusan National University, Pusan 609-735, Korea \\ ${ }^{3}$ Astrophysical Research Center for the Structure and Evolution of the Cosmos, \\ Sejong University, Seoul 143-747, Korea
}

${ }^{*}$ To whom correspondence should be addressed. E-mail: ryu@ @anopus.cnu.ac.kr

The nature and origin of turbulence and magnetic fields in the intergalactic space are important problems that are yet to be understood. We propose a scenario in which turbulent flow motions are induced via the cascade of the vorticity generated at cosmological shocks during the formation of the large scale structure. The turbulence in turn amplifies weak seed magnetic fields of any origin. Supercomputer simulations show that the turbulence is subsonic inside clusters/groups of galaxies, whereas it is transonic or mildly supersonic in filaments. Based on a turbulence dynamo model, we then estimate that the average magnetic field strength would be a few $\mu \mathrm{G}$ inside clusters/groups, approximately $0.1 \mu \mathrm{G}$ around clusters/groups, and approximately $10 \mathrm{nG}$ in filaments. Our model presents a physical mechanism that transfers the gravitation energy to the turbulence and magnetic field energies in the large scale structure of the universe. 
There is growing evidence that the intergalactic medium (IGM) is permeated with magnetic fields and is in a state of turbulence, similar to the interstellar medium within galaxies. Magnetic fields in the intracluster medium (ICM) have been measured using a variety of techniques, including observations of diffuse synchrotron emission from radio halos, inverseCompton scattered cosmic background radiation in extreme ultraviolet and hard $\mathrm{x}$-ray radiation, and Faraday rotation measure (RM). The inferred strength of the magnetic fields is on the order of $1 \mu \mathrm{G}(1,2,3)$. In the IGM outside of clusters, an upper limit of $\sim 0.1 \mu \mathrm{G}$ has been placed on the magnetic field strength of filaments, based on the observed limit of the RMs of background quasars (4, 5).

So far, signatures of turbulence have been observed only in the ICM. The analysis of the gas pressure maps of the Coma cluster revealed that pressure fluctuations are consistent with Kolmogoroff turbulence, and turbulence is likely to be subsonic with $\varepsilon_{\text {turb }} \gtrsim 0.1 \varepsilon_{\text {th }}$, where $\varepsilon_{\text {turb }}$ and $\varepsilon_{\text {th }}$ are the turbulence and thermal energy densities, respectively (6). These results agree with predictions of numerical simulations of large scale structure (LSS) formation (7, 8). Turbulence in the ICM also has been studied in RM maps of a few clusters (9, 10).

It has been suggested that cosmological shocks with Mach numbers up to $\sim 10^{4}$ and speeds up to a few thousand $\mathrm{km} \mathrm{s}^{-1}$ exist in the IGM (11, 12, 13). Such shocks result from the supersonic flow motions that are induced by the hierarchical formation of LSS in the universe. They are collisionless shocks, which form in a tenuous plasma via collective electromagnetic interactions between particles and electromagnetic fields (14). The gravitational energy released during the structure formation is transferred by these shocks to the IGM plasma in several different forms: in addition to the gas entropy, cosmic rays are produced via diffusive shock acceleration (15, 16), magnetic fields are generated via the Biermann battery mechanism (17, 7) and Weibel instability (18, 19), and vorticity is generated at curved shocks (20, 21).

In astrophysical plasmas in which charged particles are coupled to magnetic fields, turbulent 
flow motions and magnetic fields are closely related. We suggest that the turbulence in the IGM is induced by the cascade of the vorticity generated at cosmological shocks. The turbulence then amplifies the intergalactic magnetic fields (IGMFs) through the stretching of field lines, a process known as the turbulence dynamo. This scenario provides a theoretically motivated model for the evolution of the IGMFs in LSS, independent of the origin of seed fields.

There are other sources that can also provide turbulence and magnetic fields to the IGM. For instance, galactic winds can drag out the galactic magnetic fields on the order of $1 \mu \mathrm{G}$ strength into the surrounding IGM (22). The magnetic fields in the lobes of the jets from galactic black holes can also contaminate the IGM (23). Mergers of smaller objects are expected to produce turbulent motions in the ICM, which in turn amplify the existing magnetic fields (24). Those processes, although possibly important, are not topics of this study.

We first calculated the vorticity, $\vec{\omega} \equiv \vec{\nabla} \times \vec{v}$ (curl of flow velocity), in the IGM, from a numerical simulation using particle-mesh/Eulerian hydrodynamic code (25) for the formation of LSS in a cold dark matter dominated universe with a cosmological constant [supporting online material (SOM) text S1]. As shown in Fig. 1, numerous shocks exist in the LSS that are bounded by accretion shocks (11). The distribution of vorticity closely matches that of shocks, suggesting that a substantial portion of the vorticity, if not all, must have been generated at the shocks.

There is a clear trend that the vorticity is larger in hotter (Fig. 2) and denser (fig. S1) regions. As shown in the top right panel of Fig. 2 , at the present epoch, $\omega_{\text {rms }} t_{\text {age }} \sim 10$ to $30\left(\omega_{\text {rms }}\right.$, the root mean square of the vorticity; $t_{\text {age }}$, the present age of the universe) in clusters/groups (temperature $\left.T>10^{7} \mathrm{~K}\right)$ and filaments $\left(10^{5}<T<10^{7} \mathrm{~K}\right)$, whereas it is on the order of unity in sheetlike structures $\left(10^{4}<T<10^{5} \mathrm{~K}\right)$ and even smaller in voids $\left(T<10^{4} \mathrm{~K}\right.$ ) (see SOM text S2 for the temperature phases of the IGM). It increases a little with time and asymptotes after redshift $z \lesssim 1$. Because the local eddy turnover time, $t_{\text {eddy }}$, can be defined with the vorticity as 
$t_{\text {eddy }}=1 / \omega, \omega t_{\text {age }}(z)$ represents the number of eddy turnovers in the age of the universe at a given $z$. Roughly, if $\omega t_{\text {age }}$ is greater than a few, we expect there has been enough time for the vorticity to cascade down to smaller scales and for turbulence to develop in the IGM. So it is likely that turbulence is well developed in clusters/groups and filaments, but the flow is mostly non-turbulent in sheets and voids.

In our simulation the vorticity was generated either directly at curved cosmological shocks or by the baroclinity of flows. The baroclinity resulted from the entropy variation induced at shocks. Therefore, the baroclinic vorticity generation also can be attributed to the presence of cosmological shocks. Our estimates of vorticity generation by the two processes (SOM text S3) are shown with open symbols in the top right panel of Fig. 2. They agree reasonably well with the vorticity present in the simulation, although the estimates are intended to be rough. The plot indicates that the contributions from the two processes are comparable.

To estimate the energy associated with turbulence, the curl component of flow motions, $\vec{v}_{\text {curl }}$, which satisfies the relation $\vec{\nabla} \times \vec{v}_{\text {curl }} \equiv \vec{\nabla} \times \vec{v}$, is extracted from the velocity field (SOM text $\mathrm{S} 4)$. As vorticity cascades to develop into turbulence, the energy $(1 / 2) \rho v_{\text {curl }}^{2}$ ( $\rho$, gas density) is transferred to turbulent motions, so we regard it as the turbulence energy, $\varepsilon_{\text {turb }}$. As shown in Fig. $3, \varepsilon_{\text {turb }}<\varepsilon_{\text {th }}$ in clusters/groups. In particular, the mass-averaged value is $\left\langle\varepsilon_{\text {turb }} / \varepsilon_{\text {th }}\right\rangle_{\text {mass }}=0.1$ to 0.3 for $T>10^{7} \mathrm{~K}$, which is in good agreement with the observationally inferred value in cluster cores (6). The turbulence Mach number $M_{\text {turb }} \equiv v_{\text {turb }} / c_{\mathrm{s}}=\sqrt{1.8}\left(\varepsilon_{\text {turb }} / \varepsilon_{\text {th }}\right)^{1 / 2}$, where $c_{\mathrm{s}}$ is the sound speed. Therefore, overall turbulence is subsonic in clusters/groups, whereas it is transonic or mildly supersonic in filaments.

The general consensus regarding the origin of the IGMFs is that no mechanism can produce strong coherent magnetic fields in the IGM before the formation of LSS and galaxies (26). However, it is reasonable to assume that weak seed fields were created in the early universe (SOM text S5). The seed fields can be amplified by the intergalactic turbulence discussed above. 
In principle, if we were to perform magnetohydrodynamic (MHD) simulations of structure formation, the amplification of the IGMFs could be followed. In practice, however, the currently available computational resources do not allow a numerical resolution high enough to reproduce the full development of MHD turbulence in LSS (7).

In order to follow the growth of the IGMFs by the dynamo action of turbulence, we turned to a separate simulation in a controlled box. Starting with a very weak regular field, a threedimensional incompressible simulation of driven MHD turbulence was performed (SOM text S6). In the simulation, the evolution of magnetic fields goes through three stages: (i) the initial exponential growth stage, when the back-reaction of magnetic fields is negligible; (ii) the linear growth stage, when the back-reaction starts to operate; and (iii) the final saturation stage (27). Adopting the simulation result, we model the growth and saturation of magnetic energy as

$$
\phi\left(t / t_{\text {eddy }}\right)=\frac{\varepsilon_{B}}{\varepsilon_{\text {turb }}}= \begin{cases}0.04 \times \exp \left[\left(t / t_{\text {eddy }}-4\right) / 0.36\right] & \text { for } t / t_{\text {eddy }}<4 \\ (0.36 / 41) \times\left(t / t_{\text {eddy }}-4\right)+0.04 & \text { for } 4<t / t_{\text {eddy }}<45 \\ 0.4 & \text { for } t / t_{\text {eddy }}>45\end{cases}
$$

(fig. S2). Assuming that the fraction of turbulence energy governed by Eq. 1, $\phi$, is converted into the magnetic energy, we estimate the strength of the IGMFs as $B=\left[8 \pi \varepsilon_{\text {turb }} \cdot \phi\left(\omega t_{\text {age }}\right)\right]^{1 / 2}$. Here the values of $\omega$ and $\varepsilon_{\text {turb }}$ are calculated locally from the structure formation simulation.

The resulting IGMFs follows the cosmic web of matter distribution as shown in Fig. 4 (and in fig. S3). On average the IGMFs are stronger in hotter (Fig. 2) and denser (fig. S1) regions in our model. The strength of the IGMFs is $B \gtrsim 1 \mu \mathrm{G}$ inside clusters/groups (the massaveraged value for $T>10^{7} \mathrm{~K}$ ), $\sim 0.1 \mu \mathrm{G}$ around clusters/groups (the volume-averaged value for $T>10^{7} \mathrm{~K}$ ), and $\sim 10 \mathrm{nG}$ in filaments at present (bottom right panel of Fig. 2) (see SOM text S7 for the numerical convergence of the estimation). These values agree with the observed field strengths discussed earlier. They also agree with the previous study (7), in which the magnetic field strength in clusters was estimated to be a few $\mu \mathrm{G}$, based on a kinetic theory. The IGMFs should be much weaker in sheetlike structures and voids. But as noted above, turbulence 
is not fully developed in such low density regions, so our model is not adequate to predict the field strength there. For each temperature phase, the IGMFs are stronger in the past, because the gas density is higher. However, the IGMFs averaged over the entire computational volume are weaker in the past because the fraction of strong field regions is smaller.

While being amplified, magnetic fields become coherent through the inverse cascade (27). The coherence scale of magnetic fields in fully developed turbulence is expected to be several times smaller than the driving scale, that is, the scale of dominant eddies (SOM text S8). In the IGM outside of clusters, the curvature radius of typical cosmological shocks is approximately a couple of Mpc (11) (fig. S4), which should represent a characteristic scale of dominant eddies. The coherence length of the IGMFs there is then expected to be several $100 \mathrm{kpc}$. On the other hand, the scale height of the ICM is several $100 \mathrm{kpc}$. If it corresponds to the scale of the dominant eddies, the coherence length in the ICM is expected to be $\sim 100 \mathrm{kpc}$ or so.

Our model can predict the RMs owing to the IGMFs, which may be tested in future observations with Low Frequency Array and Square Kilometer Array (28). Also, our model IGMFs can be employed in the study of the propagation of ultra-high-energy cosmic rays, which is crucial to search for astrophysical accelerators of such high energy particles (29). 


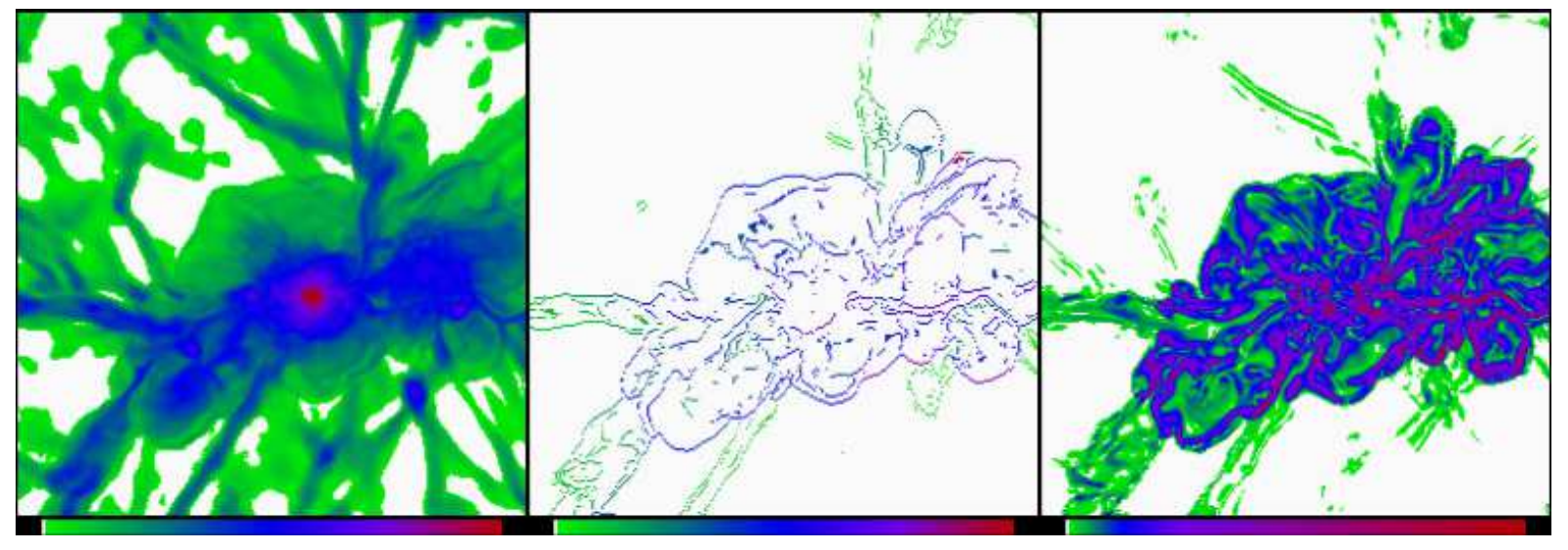

Fig. 1. Two-dimensional images showing gas density $\rho$ in a logarithmic scale (left), locations of shocks with color-coded shock speed $v_{\text {shock }}$ (middle), and magnitude of vorticity $\omega t_{\text {age }}$ (right), around a cluster complex of $\left(25 h^{-1} \mathrm{Mpc}\right)^{2}$ area at present $(z=0)$. Here, $h$ is the Hubble constant in units of $100 \mathrm{~km} \mathrm{~s}^{-1} \mathrm{Mpc}^{-1}$. The complex includes a cluster of $\mathrm{x}$-ray emissionweighted temperature $T_{x} \approx 3.3 \mathrm{keV}$. Color codes for each panel are (left) $\rho /\langle\rho\rangle$ from $10^{-1}$ (green) to $10^{4}$ (red); (middle) $v_{\text {shock }}$ from 15 (green) to $1,800 \mathrm{~km} \mathrm{~s}^{-1}$ (red); and (right) $\omega t_{\text {age }}$ from 0.5 (green) to 100 (red). 


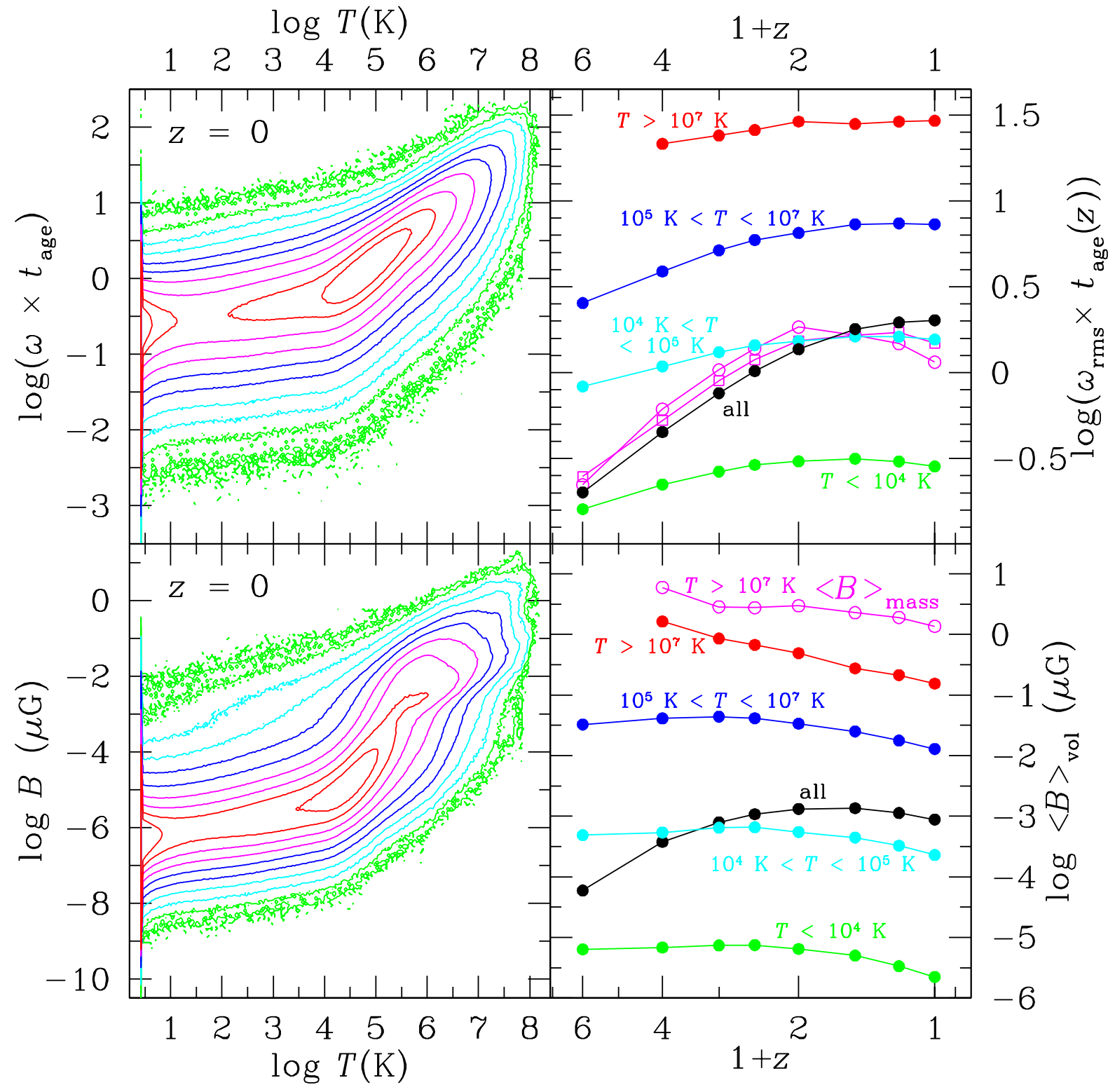

Fig. 2. (Left) Volume fraction with given temperature and vorticity magnitude (top left) and temperature and magnetic field strength (bottom left) at present. (Right) Time evolution of the root mean square of the vorticity (top right) and the volume-averaged magnetic field strength (bottom right) for four temperature phases of the IGM and for all the gas as a function of redshift $z$. Magenta symbols in the top right panel are our estimates of the vorticity generated directly at curved shocks (open circles) and by the baroclinity of flows (open squares). Magenta open circles in the bottom right panel show the mass-averaged magnetic field strength for $T>10^{7}$ K. 




Fig. 3. Ratio of turbulence to thermal energies as a function of temperature at present. The values shown are volume-averaged and mass-averaged over temperature bins. 


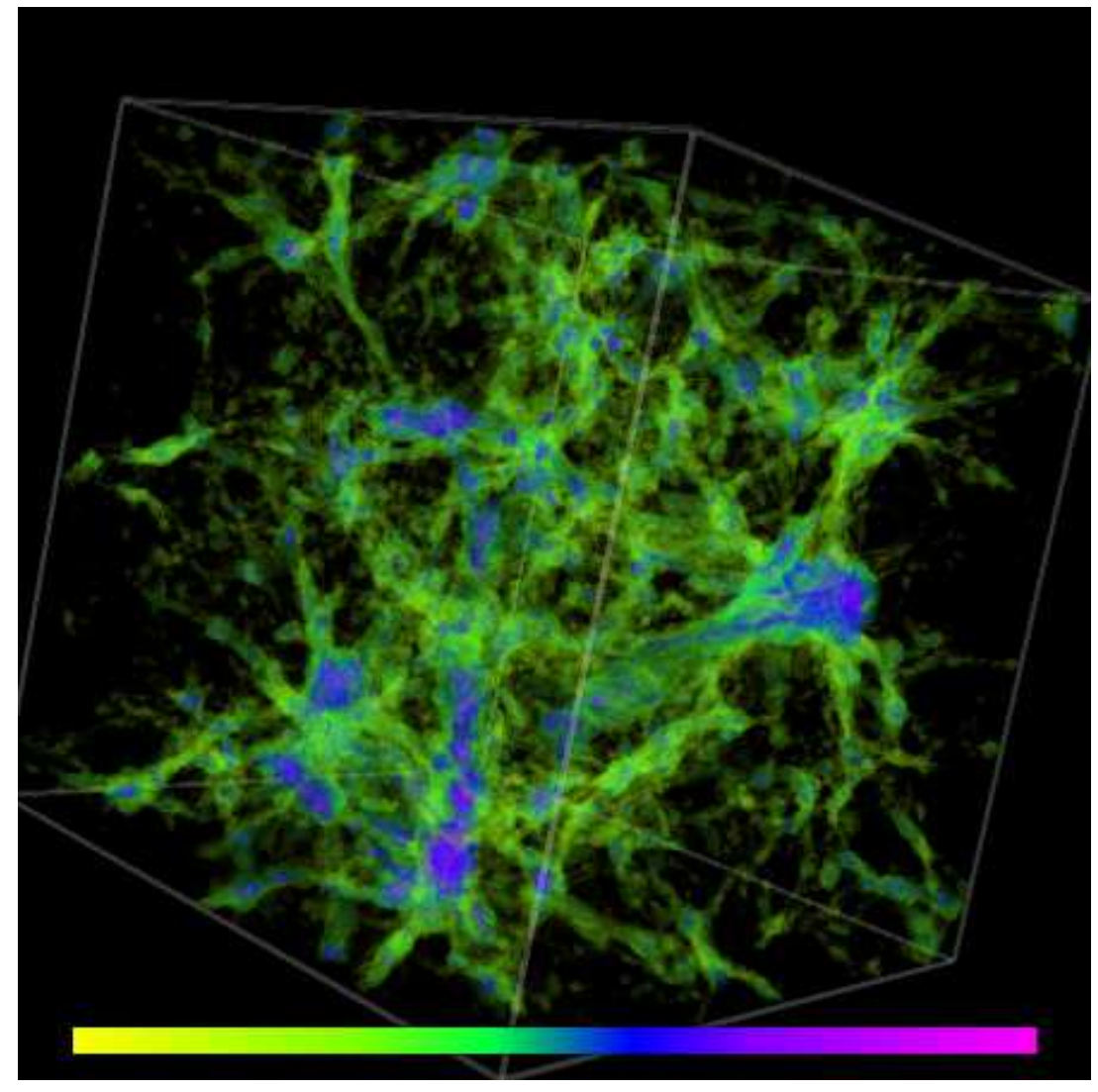

Fig. 4. Volume-rendering image showing the logarithmically scaled magnetic field strength at $z=0$ in the whole computational box of $\left(100 h^{-1} \mathrm{Mpc}\right)^{3}$ volume. Color codes the magnetic field strength from $0.1 \mathrm{nG}$ (yellow) to $10 \mu \mathrm{G}$ (magenta). The colors were chosen so that clusters and groups show as magenta and blue and filaments as green. 


\section{References and Notes}

1. C. L. Carilli, G. B. Taylor, Annu. Rev. Astron. Astrophys. 40, 319 (2002)

2. F. Govoni, L. Feretti, Int. J. Mod. Phys. D 13, 1549 (2004)

3. T. E. Clarke, P. P. Kronberg, H. Böhringer, Astrophys. J. 547, L111 (2001)

4. D. Ryu, H. Kang, P. L. Biermann, Astron. Astrophys. 335, 19 (1998)

5. Y. Xu, P. P. Kronberg, S. Habib, Q. W. Dufton, Astrophys. J. 637, 19 (2006)

6. P. Schuecker, A. Finoguenov, F. Miniati, H. Böhringer, U. G. Briel, Astron. Astrophys. 426, 387 (2004)

7. R. M. Kulsrud, R. Cen, J. P. Ostriker, D. Ryu, Astrophys. J. 480, 481 (1997)

8. D. Nagai, A. Vikhlinin, A. V. Kravtsov, Astrophys. J. 655, 98 (2007)

9. M. Murgia et al., Astron. Astrophys. 424, 429 (2004)

10. C. Vogt, T. Enßlin, Astron. Astrophys. 434, 67 (2005)

11. D. Ryu, H. Kang, E. Hallman, T. W. Jones, Astrophys. J. 593, 599 (2003)

12. C. Pfrommer, V. Springel, T. A. Enßlin, M. Jubelgas, Mon. Not. Roy. Astron. Soc. 367, 113 (2006)

13. H. Kang, D. Ryu, R. Cen, J. P. Ostriker, Astrophys. J. 669, 729 (2007)

14. K. B. Quest, J. Geophys. Res. 93, 9649 (1988)

15. A. R. Bell, Mon. Not. Roy. Astron. Soc. 182, 147 (1978)

16. R. D. Blandford, J. P. Ostriker, Astrophys. J. 221, L29 (1978) 
17. L. Biermann, Z. Naturforsch 5a, 65 (1950)

18. E. S. Weibel, Phys. Rev. Lett. 2,83 (1959)

19. M. V. Medvedev, L. O. Silva, M. Kamionkowski, Astrophys. J. 642, L1 (2006)

20. J. Binney, Mon. Not. Roy. Astron. Soc. 168, 73 (1974)

21. G. Davies, L. M. Widrow, Astrophys. J. 540, 755 (2000)

22. H. J. Völk, A. M. Atoyan, Astropart. Phys. 11, 73 (1999).

23. P. P. Kronberg, Q. W. Dufton, H. Li, S. A. Colgate, Astrophys. J. 560, 178 (2001)

24. K. Subramanian, K., A. Shukurov, N. E. L. Haugen, Mon. Not. Roy. Astron. Soc. 366, 1437 (2006).

25. D. Ryu, J. P. Ostriker, H. Kang, R. Cen, Astrophys. J. 414, 1 (1993)

26. R. M. Kulsrud, E. G. Zweibel, Rep. Prog. Phys. 71, 046901 (2008)

27. J. Cho, E. T. Vishniac, Astrophys. J. 538, 217 (2000)

28. B. M. Gaensler, R. Beck, L. Feretti, New Astron. Rev. 48, 1003 (2004)

29. S. Das, H. Kang, D. Ryu, J. Cho Astrophys. J. 681, in press (2008)

30. This work was supported by KICOS through grant K20702020016-07E0200-01610 provided by MOST. The work of D.R. was also supported by KOSEF through the grant of basic research program R01-2007-000-20196-0. The work of H.K. and S.D. was also supported by KOSEF through ARCSEC. The work of J.C. was also supported by KRF through grant KRF-2006-331-C00136 funded by the Korean Government. 


\section{Supporting Online Material}

\section{Materials and Methods}

\section{S1. Simulation of large scale structure formation}

The simulation used to estimate the vorticity in the intergalactic medium (IGM) is for a $\Lambda \mathrm{CDM}$ universe with the following values of cosmological parameters: $\Omega_{B M}=0.043, \Omega_{D M}=0.227$, $\Omega_{\Lambda}=0.73, h \equiv H_{0} /(100 \mathrm{~km} / \mathrm{s} / \mathrm{Mpc})=0.7$, and $\sigma_{8}=0.8$. It was performed using a PM/Eulerian hydrodynamic code for the formation of large scale structure (LSS) (S1), in a cubic region of comoving volume $\left(100 h^{-1} \mathrm{Mpc}\right)^{3}$ with $1024^{3}$ grid zones for gas and gravity and $512^{3}$ particles for dark matter, allowing a spatial resolution of $\Delta l=97.7 \mathrm{kpc}$. The simulation is adiabatic in the sense that it does not include radiative cooling, galaxy/star formation, feedbacks from galaxies/stars, and reionization of the IGM. Although these processes play important roles in determining the physical conditions mainly in cluster cores and voids, it was shown that they do not affect significantly the global properties and dissipations of cosmological shocks (S2, S3).

\section{S2. Phases of the IGM}

The intergalactic gas is heated mainly by cosmological shocks (S4, S5). It was suggested that the IGM can be classified roughly into four phases according to gas temperature: the hot gas with $T>10^{7} \mathrm{~K}$ mostly inside and around clusters/groups, the warm-hot intergalactic medium (WHIM) with $T=10^{5}-10^{7} \mathrm{~K}$ found mostly in filaments, the low temperature WHIM with $T=10^{4}-10^{5} \mathrm{~K}$ distributed mostly as sheetlike structures, and the diffuse gas with $T<10^{4} \mathrm{~K}$ residing mostly in voids (S5, S6). In particular, the intracluster medium (ICM) refers to the hottest phase of the IGM inside clusters that is observable in x-ray.

\section{S3. Vorticity generation}

Vorticity can be generated directly at curved shocks and by the baroclinity of flows. For uniform 
upstream flow, the vorticity produced behind curved shock surface is

$$
\vec{\omega}_{\mathrm{cs}}=\frac{\left(\rho_{2}-\rho_{1}\right)^{2}}{\rho_{1} \rho_{2}} K \vec{U}_{1} \times \hat{n},
$$

where $\rho_{1}$ and $\rho_{2}$ are the upstream and downstream gas densities, respectively, $\vec{U}_{1}$ is the upstream flow velocity in the shock rest frame, $K$ is the curvature tensor of the shock surface, and $\hat{n}$ is the unit vector normal to the surface (S7). If isopycnic surfaces (constant density surfaces) do not coincide with isobaric surfaces, vorticity is generated with the rate given by

$$
\dot{\vec{\omega}}_{\mathrm{bc}}=\frac{1}{\rho^{2}} \vec{\nabla} \rho \times \vec{\nabla} p
$$

Rough estimates of the vorticity generations by the two processes in the simulation of LSS formation are made as follows. Considering the generation is a random walk process,

$$
(\dot{\omega} \Delta t)_{\mathrm{rms}}\left(\frac{t_{\text {age }}(z)}{\Delta t}\right)^{1 / 2}
$$

is calculated. Here $\Delta t$ is the duration of coherent generation, and so $t_{\text {age }}(z) / \Delta t$ is the number of random walks during the age of the universe at redshift $z$. For the direct generation at cosmological shocks,

$$
\dot{\omega}_{\mathrm{cs}} \sim \frac{\left(\rho_{2}-\rho_{1}\right)^{2}}{\rho_{1} \rho_{2}} \frac{U_{n 1} U_{n 2}}{R} \frac{g}{\Delta l}
$$

is used. Here $R$ is the curvature radius of the shocks, $U_{n 1}$ and $U_{n 2}$ are the flow speeds normal to the shocks in upstream and downstream, $\Delta l$ is the grid size, and $g$ takes account of all other factors such as geometric ones. For the baroclinic vorticity generation, $\dot{\omega}_{\mathrm{bc}}$ from Eq. (S2) is used. In the estimations, the distribution and property of shocks and flows from the simulation are used. Also $\Delta t$ is taken to be $10^{8}$ yrs, which is a typical dynamical time, e.g., the sound crossing time in cluster cores. The comoving value of $\langle R\rangle \sim 1 h^{-1} \mathrm{Mpc} /(1+z)$, and $\langle g\rangle \sim 1$ are taken. Note that $1 h^{-1} \mathrm{Mpc}$ is the typical scale of nonlinear structures at present. The factor $1 /(1+z)$ is included because the average curvature radius of cosmological shocks is smaller in 
the past even in the comoving frame (S8). In Fig. 2 the quantities normalized with $1 / t_{\text {age }}(z)$ are shown.

\section{S4. Decomposition of flow velocity}

The flow velocity can be decomposed into

$$
\vec{v}=\vec{v}_{\text {div }}+\vec{v}_{\text {curl }}+\vec{v}_{\text {unif }}
$$

where the divergence and curl components are defined as $\vec{\nabla} \cdot \vec{v}_{\text {div }} \equiv \vec{\nabla} \cdot \vec{v}$ and $\vec{\nabla} \times \vec{v}_{\text {curl }} \equiv \vec{\nabla} \times \vec{v}$, respectively. That is, $\vec{v}_{\text {div }}$ is associated to compressional motions, while $\vec{v}_{\text {curl }}$ to incompressible shear motions. Here $\vec{v}_{\text {unif }}$ is the component uniform across the computational box, whose magnitude is much smaller than the other two components. The decomposition is calculated exactly in Fourier space. We note with the above decomposition, locally

$$
\vec{v}_{\text {div }} \cdot \vec{v}_{\text {curl }} \neq 0 \quad \text { so } \quad \frac{1}{2} v^{2} \neq \frac{1}{2}\left(v_{\text {div }}^{2}+v_{\text {curl }}^{2}\right)
$$

However, globally

$$
\int_{\text {box }} \vec{v}_{\text {div }} \cdot \vec{v}_{\text {curl }} d^{3} \vec{x}=0 \quad \text { so } \quad \int_{\text {box }} \frac{1}{2} v^{2} d^{3} \vec{x}=\int_{\text {box }} \frac{1}{2}\left(v_{\text {div }}^{2}+v_{\text {curl }}^{2}\right) d^{3} \vec{x} .
$$

\section{S5. Seed magnetic fields}

A number of mechanisms that can create seeds field in the early universe have been suggested. Besides various inflationary and string theory mechanisms, the most popular astrophysical mechanism is the Biermann battery $(S 9)$. It was discussed in the context of cosmological shocks (S10) and ionization fronts (S11), and shown to build up weak fields of strength up to $10^{-20} \mathrm{G}$ by $z \sim$ a few.

At cosmological shocks, in addition, Weibel instability can operate and produce magnetic fields up to the level of $\varepsilon_{B} \sim 10^{-3} \varepsilon_{\mathrm{sh}}$ (S12), and streaming cosmic rays accelerated by the shocks can amplify weak upstream magnetic fields up to the level of $\varepsilon_{B} \sim(1 / 2)\left(U_{1} / c\right) \varepsilon_{\mathrm{CRs}}$ 
via non-resonant growing mode (S13). Here $\varepsilon_{\mathrm{sh}}$ is the energy density of upstream flow, and $\varepsilon_{B}$ and $\varepsilon_{\mathrm{CRs}}$ are the energy densities of downstream magnetic fields and CRs. With $U_{1} / c \sim 10^{-3}$ for cosmological shocks $(S 2, S 3)$, these processes can potentially produce stronger seed fields, although the coherence length of the resulting fields is expected to very small and microscopic.

\section{S6. Simulation of MHD turbulence}

First we note there are at least two distinct types of magnetohydrodynamic (MHD) turbulence: one with strong regular fields and the other with weak/zero regular fields. The former has been described successfully with the nonlinear interactions of Alfvén waves (S14). The latter is mainly hydrodynamic in large scales, but is modified by dynamically important magnetic fields in small scales $(S 15, S 16)$. It is the latter that is relevant to this work.

Incompressible, driven turbulence was simulated initially with a very weak regular field. A pseudo-spectral code (S15) was used, employing hyper-viscosity and hyper-resistivity with the Prandtl number of unity. The advantages of this numerical approach include virtually zero intrinsic numerical viscosity and resistivity, and the maximized inertial range. The simulation was performed with a numerical resolution of $256^{3}$ collocation points. The turbulence was driven at the scale of $L_{\text {driving }} \sim(1 / 2) L_{\text {box }}$, where $L_{\text {box }}$ is the computational box size. The driving strength was set so that the total turbulence energy becomes $\varepsilon_{\text {turb }} \equiv \varepsilon_{\text {kin }}+\varepsilon_{B} \sim 1$ at saturation. Initially $\varepsilon_{B}=10^{-6}$, but the evolution is not sensitive to the initial field strength as long as it is sufficiently weak (S16). The left panel of Fig. S2 shows the time evolution of kinetic and magnetic energies. Here, the eddy turnover time is defined with the vorticity at driving scale at saturation, $t_{\text {eddy }} \equiv 1 / \omega_{\text {driving }}$.

We made the incompressible simulation, because compressible simulations need much higher resolution to achieve the same growth rate and magnetic field strength at saturation. In addition, due to large numerical dissipation, controlling viscosity and resistivity is not trivial in compressible simulations. Nevertheless, in order to show the general behavior of turbulence 
dynamo seen in our incompressible simulation remains the same in compressible regime, we performed compressible simulations of driven MHD turbulence, using a code based on the third-order essentially non-oscillatory (ENO) upwind scheme (S17). This is one of the numerical schemes for MHD with least numerical dissipation. Different numerical resolutions of $8^{3}$ to $216^{3}$ grid zones were used. But otherwise, the initial conditions, the driving of turbulence, and hyper-viscosity and hyper-resistivity with the Prandtl number of unity were the same as in the incompressible simulation. We only considered the case with the Mach number $M_{s} \sim 1$, which is most relevant to this work. We note that turbulence dynamo can be suppressed in highly supersonic flows (S18).

Fig. S5 compares the growth rate (left panel) and the magnetic energy at saturation (right panel) from compressible simulations with those from incompressible simulation. Two points are clear from the figure: (1) the growth rate is slower in compressible simulations, but the pattern follows that in incompressible turbulence, and (2) compressible simulation would need more than $1000^{3}$ grid zones to achieve the saturated magnetic field strength of incompressible simulation. It also demonstrates why it is impossible to reproduce the full development of MHD turbulence in the simulations of LSS formation with the currently available computational resources.

\section{S7. Convergence of our results}

In order to test the numerical convergence of our estimation for the strength of the intergalactic magnetic fields (IGMFs), we repeated the same analysis for the simulations of LSS formation with lower numerical resolutions of $64^{3}$ to $512^{3}$ grid zones. Except the resolution, they are the same simulations as that in $\S \mathrm{S} 1$, in the sense that they have the same box size, the same realization of initial conditions, and the same physics included. Fig. S6 shows the volume-averaged magnetic field strength with different resolutions. The averaged strength around clusters/groups with $T>10^{7} \mathrm{~K}$ is well converged at the resolution of $1024^{3}$. On the other hand the averaged 
strength in filaments with $10^{5}<T<10^{7} \mathrm{~K}$ would be underestimated. The converged value may be $\sim 2-3$ times larger than our estimate with the resolution of $1024^{3}$.

\section{S8. Coherence length}

The consideration of the coherence length of turbulent magnetic fields raises the issues of the scale of energy equipartition and the magnetic field structure at the scale. The right panel of Fig. S2 shows the power spectra for flow velocity and magnetic fields at a time of saturation from the simulation in $\S S 6$. The energy equipartition occurs at $\sim(1 / 2.5) L_{\text {driving, }}$, which is close to the

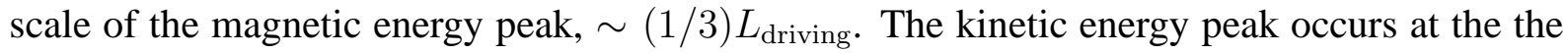

driving scale, $L_{\text {driving. }}$. Above the equipartition scale the flow structure is nearly isotropic, while below it both the flow and magnetic field structures are anisotropic and the eddies are stretched along the local magnetic field lines (S15). On the other hand, the magnetic field has most power

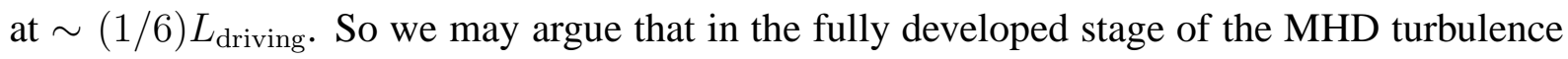
considered here, the coherence length of magnetic fields would be several times smaller than the driving scale, the scale of dominant eddies.

\section{Additional Figures Used in Manuscript}

Fig. S1 shows the correlation between the magnitude of vorticity and the gas density (left panel) and the strength of magnetic fields and the gas density (right panel) at present.

Fig. S3 shows the spatial distribution of our model IGMFs around a cluster complex and along a filament. In the left image, in addition to the main cluster of $T_{x} \approx 3.3 \mathrm{keV}$, a group has $B \sim \mathrm{a}$ few $\mu \mathrm{G}$. They are surrounded by a broad region of $B \sim 10 \mathrm{nG}$. In the right image, groups with $B \sim \mu \mathrm{G}$ are distributed along the filament with $B \sim 10 \mathrm{nG}$.

Fig. S4 shows the power spectra for the flow velocity and its curl and divergence components (§S4) at present from the simulation of LSS formation in $\S S 1$. At long wavelengths, the ampli- 
tude of perturbations are small, so that the linear theory applies. That is, $P_{\text {curl }}(k) \rightarrow 0$ as $k \rightarrow 0$, while $P_{\text {div }}(k)$ follows the analytic theory expectation, $P_{\text {div }}(k) \sim k^{-1}$. For wavelengths smaller than a few $h^{-1} \mathrm{Mpc}$, nonlinearities dominate, and we see that $P_{\text {curl }}(k) \gtrsim P_{\text {div }}(k) . \quad P_{\text {curl }}(k)$ peaks at $\sim 5 h^{-1} \mathrm{Mpc}$, and for values of $k$ somewhat larger than the peak wavenumber, the spectrum follows a power law of $k^{-5 / 3}$, the Kolmogorov spectrum. $P_{\text {curl }}(k)$ has most power at $\sim 2 h^{-1} \mathrm{Mpc}$, that indicates the typical scale of nonlinear structures in the simulation. 


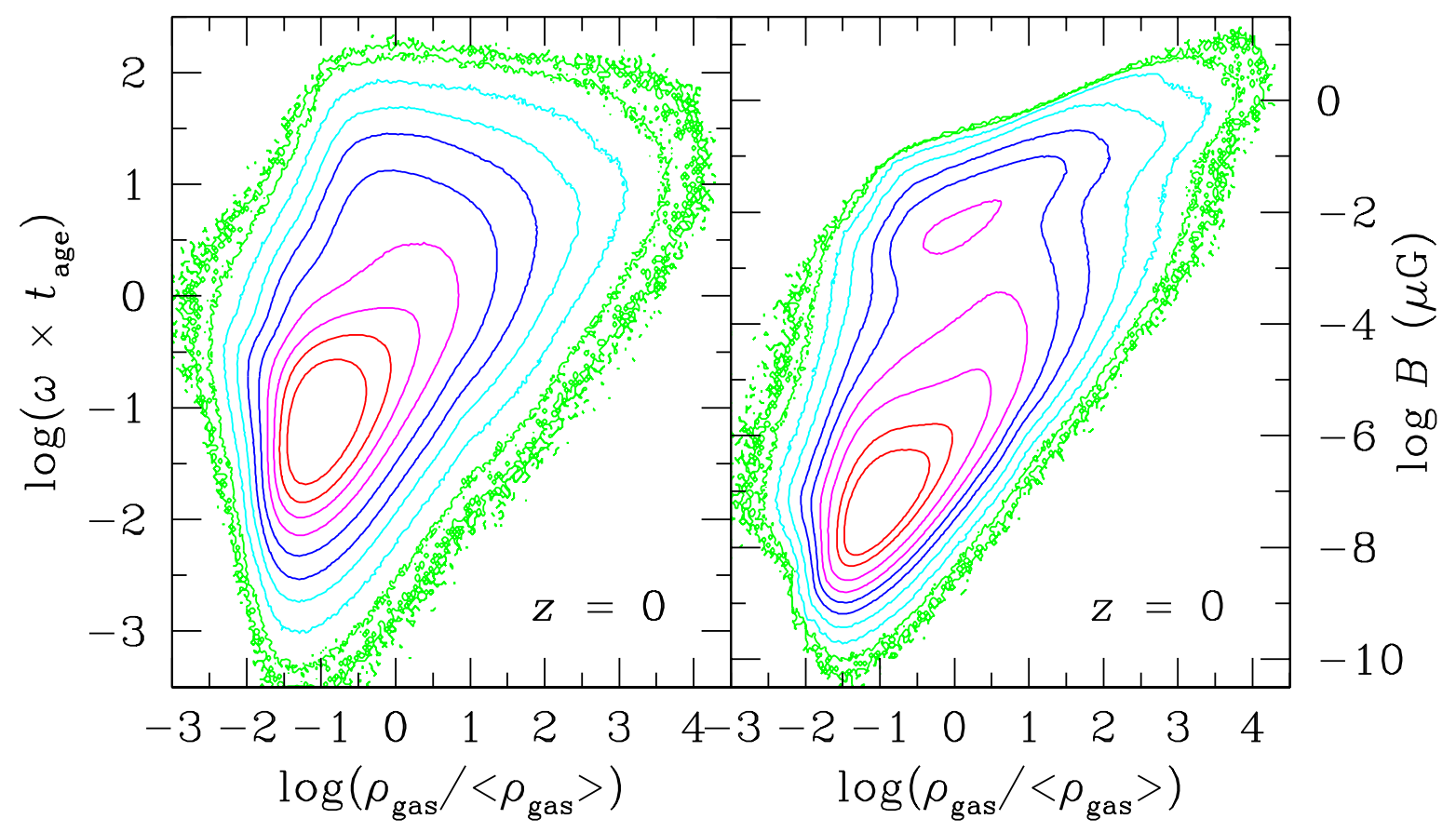

Fig. S1. Volume fraction with given gas density and vorticity magnitude (left panel) and gas density and magnetic field strength (right panel) at present. 


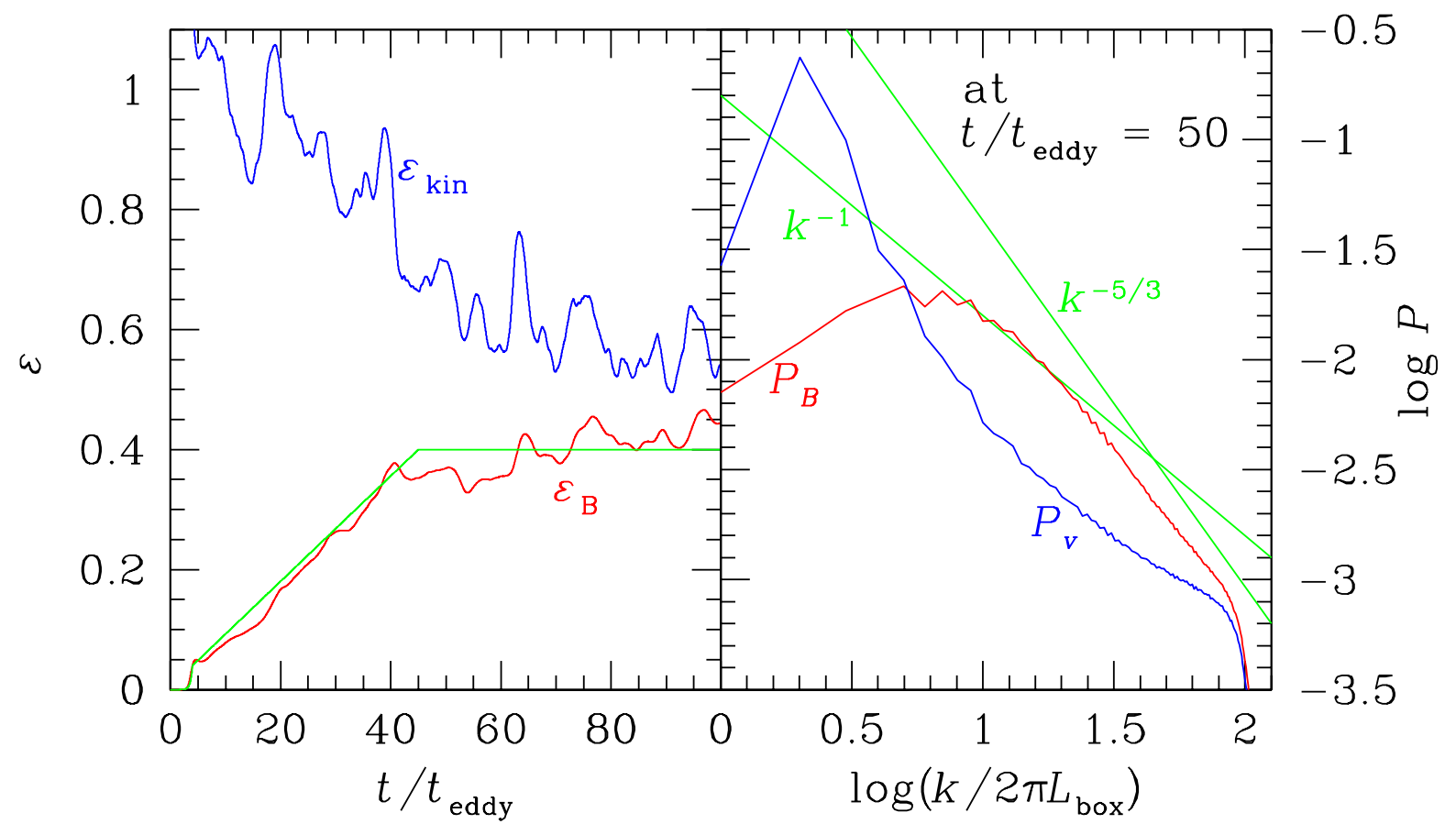

Fig. S2. Left panel: Time evolution of kinetic and magnetic energies in a three-dimensional, incompressible simulation of driven MHD turbulence with very weak initial magnetic field. The green lines show our fitting for the growth and saturation of magnetic energy. Right panel: Power spectra for flow velocity, $P_{v}$, and magnetic fields, $P_{B}$, at a time of saturation. Two straight lines of slopes $-5 / 3$ and -1 are also drawn for comparison. 


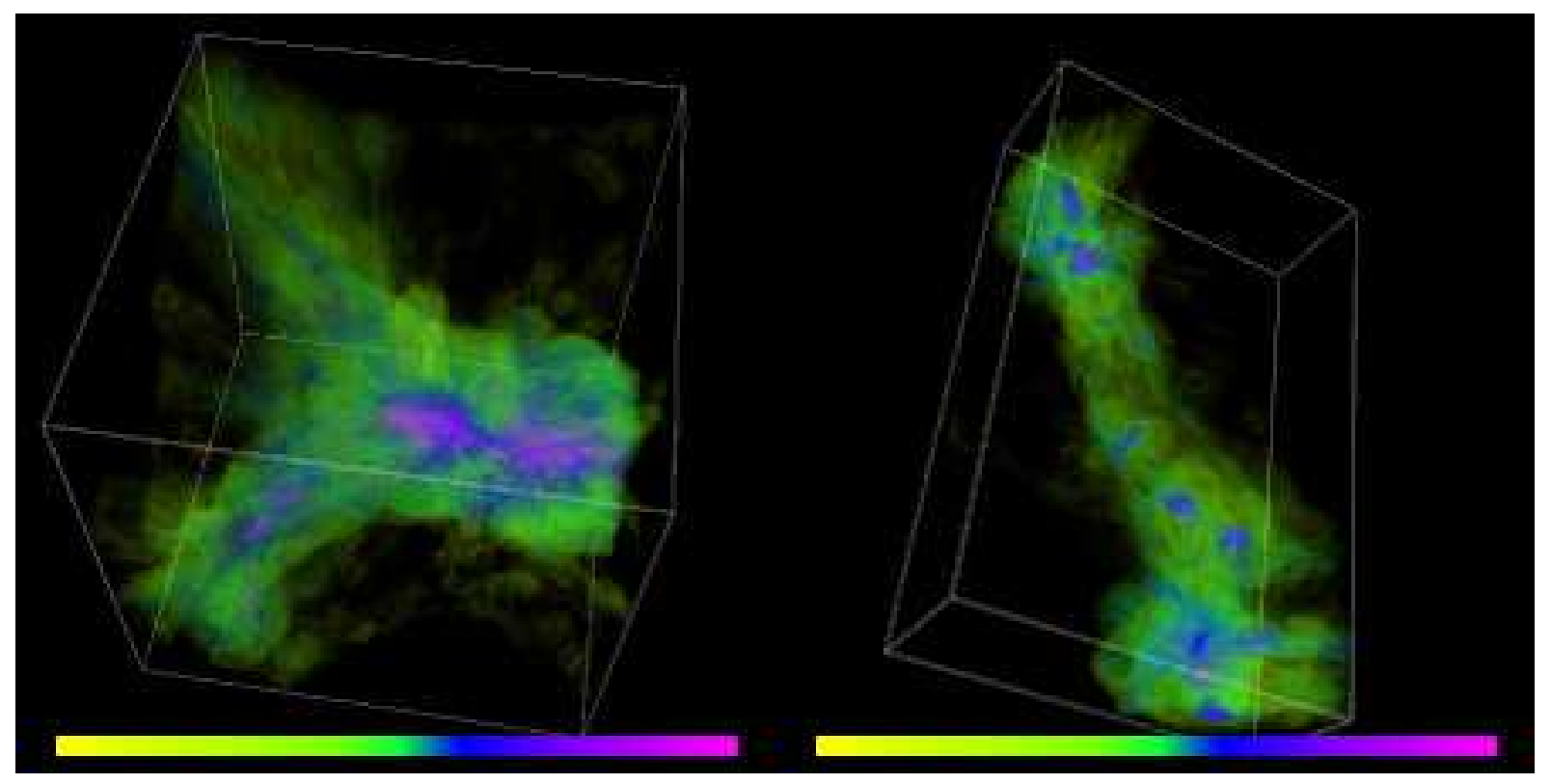

Fig. S3. Volume rendering images showing logarithmically scaled magnetic field strength at $z=0$ in a volume of $\left(25 h^{-1} \mathrm{Mpc}\right)^{3}$ around the same cluster complex as shown in Fig. 1 (left panel) and in a volume of $25 \times 15.6 \times 6.25\left(h^{-1} \mathrm{Mpc}\right)^{3}$ which includes a number of groups along a filament (right panel). As in Fig. 4, color codes the magnetic field strength from $0.1 \mathrm{nG}$ (yellow) to $10 \mu \mathrm{G}$ (magenta). Clusters and groups are shown with magenta and blue while filaments with green. 




Fig. S4. Power spectra, $\int P_{v} d k=\left\langle(1 / 2) v^{2}\right\rangle$, for flow velocity and its curl and divergence components at present in the simulation of LSS formation. Two straight lines of slopes $-5 / 3$ and -1 are also drawn for comparison. 


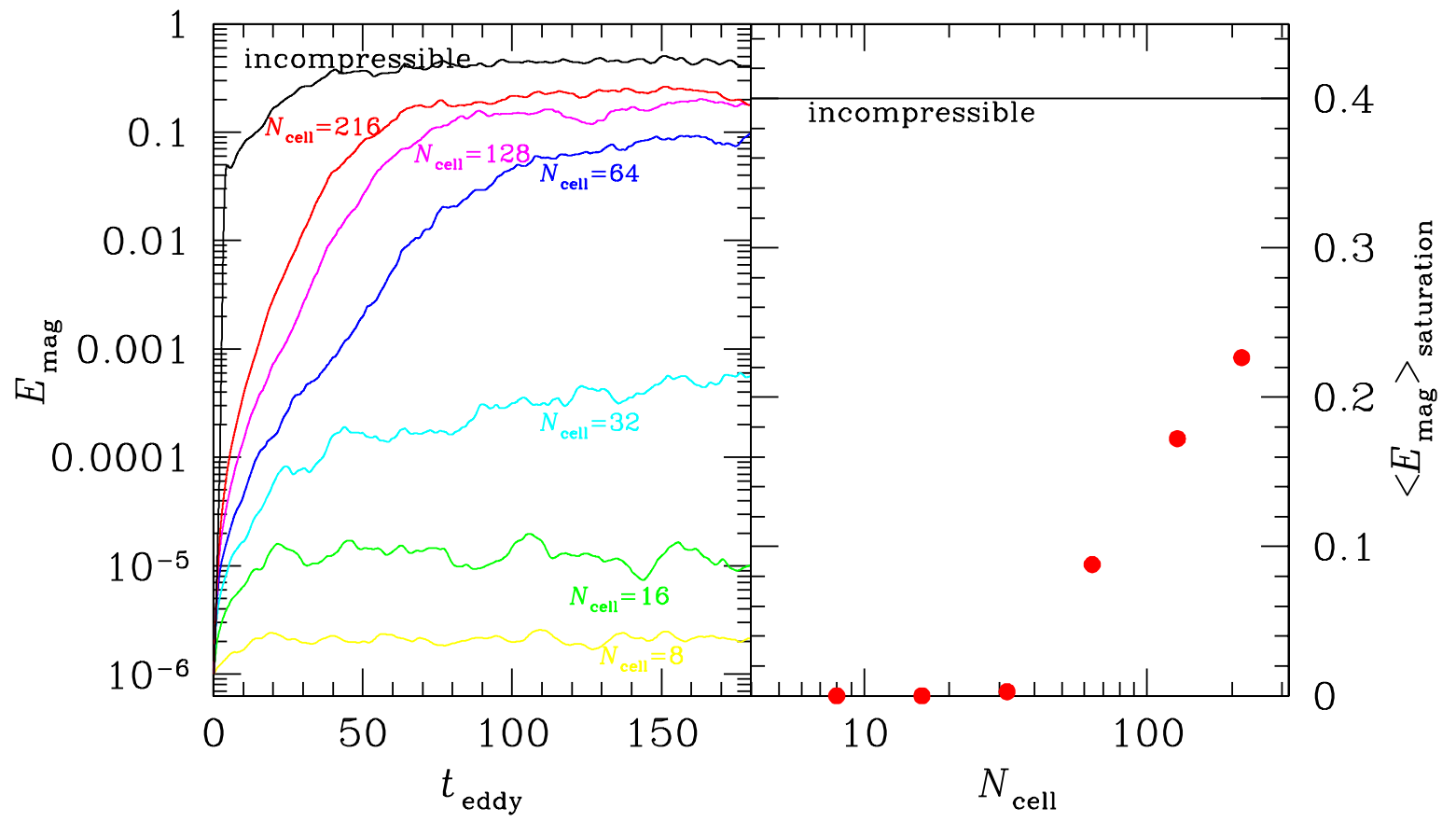

Fig. S5. Left panel: Time evolution of magnetic energy in three-dimensional, compressible simulations of driven MHD turbulence with very weak initial magnetic field. For comparison, the evolution in incompressible simulation shown in Fig. S3 is also plotted. Right panel: Magnetic energy at saturation in compressible simulations with different resolutions of $N_{\text {cell }}^{3}$. The value in incompressible simulation is marked with solid line. 


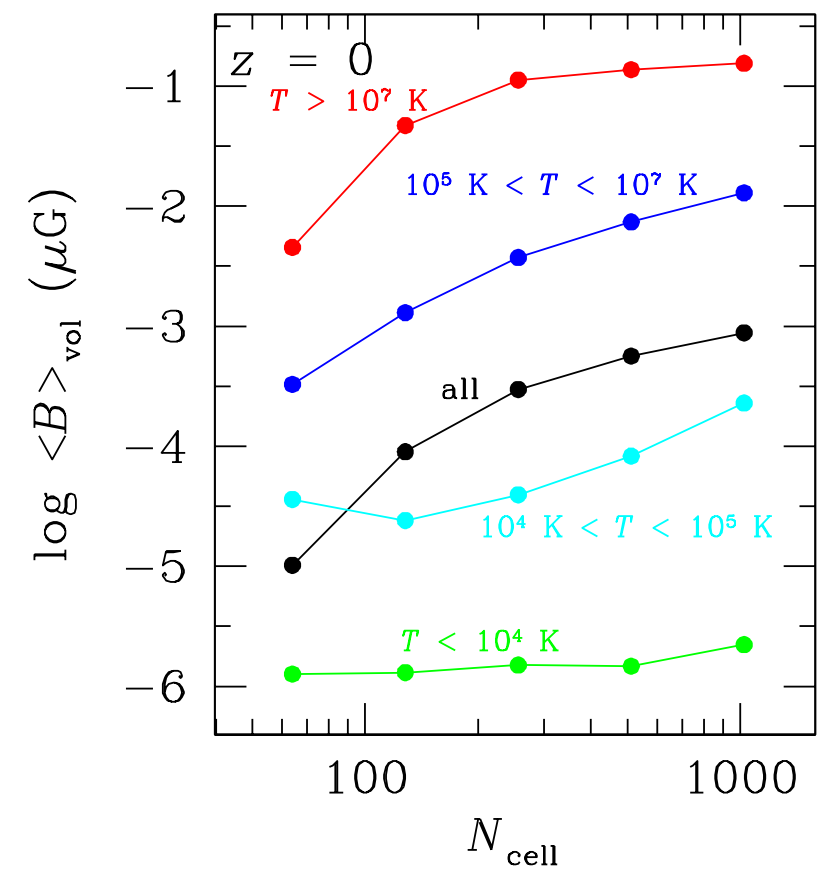

Fig. S6. Volume-averaged magnetic field strength at present for four temperature phases of the IGM and for all the gas from structure formation simulations with different resolutions of $N_{\text {cell }}^{3}$. 


\section{References}

S1. D. Ryu, J. P. Ostriker, H. Kang, R. Cen, Astrophys. J. 414, 1 (1993)

S2. D. Ryu, H. Kang, E. Hallman, T. W. Jones, Astrophys. J. 593, 599 (2003)

S3. H. Kang, D. Ryu, R. Cen, J. P. Ostriker, Astrophys. J. 669, 729 (2007)

S4. R. Cen, J. P. Ostriker, Astrophys. J. 514, 1 (1999)

S5. R. Davé et al. Astrophys. J. 552, 473 (2001)

S6. H. Kang, D. Ryu, R. Cen, D. Song, Astrophys. J. 620, 21 (2005)

S7. W. D. Hayes, J. Fluid Mech. 2, 595 (1957)

S8. D. Ryu, H. Kang J. Korean Astron. Soc. 37, 477 (2004)

S9. L. Biermann, Z. Naturforsch 5a, 65 (1950)

S10. R. M. Kulsrud, R. Cen, J. P. Ostriker, D. Ryu, Astrophys. J. 480, 481 (1997)

S11. N. Y. Gnedin, A. Ferrara, E. G. Zweibel, Astrophys. J. 539, 505 (2000)

S12. M. V. Medvedev, L. O. Silva, M. Kamionkowski, Astrophys. J. 642, L1 (2006)

S13. A. R. Bell, Mon. Not. Roy. Astron. Soc. 353, 550 (2004)

S14. P. Goldreich, S. Sridhar, Astrophys. J. 438, 763 (1995)

S15. J. Cho, E. T. Vishniac, Astrophys. J. 538, 217 (2000)

S16. J. Cho, E. T. Vishniac, A.Beresnyak, A. Lazarian, D. Ryu, Astrophys. J. submitted (2008)

S17. J. Cho, A. Lazarian, Phys. Rev. Lett. 88, 245001 (2002) 
S18. N. E. L. Haugen, A. Brandenburg, A. J. Mee, Mon. Not. Roy. Astron. Soc. 353, 947 (2004) 